\title{
Two-Stage Transmitter Precoding Based on Data-Driven Code-Hopping and Partial Zero Forcing Beamforming for MC-CDMA Communications
}

\author{
Christos Masouros, Student Member, IEEE, and Emad Alsusa, Senior Member, IEEE
}

\begin{abstract}
This paper proposes a hybrid transmission technique based on adaptive code-to-user allocation and linear precoding for the downlink of phase shift keying (PSK) based multi-carrier code division multiple access (MC-CDMA) systems. The proposed scheme is based on the separation of the instantaneous multiple access interference (MAI) into constructive and destructive components taking into account the dependency on both the channel variation and the instantaneous symbol values of the active users. The first stage of the proposed technique is to adaptively distribute the available spreading sequences to the users on a symbol-by-symbol basis in the form of codehopping with the objective to steer the users' instantaneous crosscorrelations to yield a favourable constructive to destructive MAI ratio. The second stage is to employ a partial transmitter based zero forcing (ZF) scheme specifically designed for the exploitation of constructive MAI. The partial $\mathrm{ZF}$ processing decorrelates destructive interferers, while users that interfere constructively remain correlated. This results in a signal to interference-plus-noise ratio (SINR) enhancement without the need for additional power-per-user investment. It will be shown in the results section that significant bit error rate (BER) performance benefits can be achieved with this technique.
\end{abstract}

Index Terms-Adaptive signal processing, code division multiaccess, decorrelation, interference suppression, precoding.

\section{INTRODUCTION}

$\mathbf{T}$ HE nature of the communication channel in realistic wireless systems makes interuser orthogonality unattainable in cellular MC-CDMA systems [1,2], even when orthogonal spreading sequences are employed. The resulting MAI can greatly degrade performance. For the downlink of cellular systems, two popular mechanisms for alleviating this impediment are by designing and optimizing the codes according to the specific channel and by applying linear transformations on the data to be transmitted so that the received symbols appear orthogonal at the receiver. Both these alternatives have been well investigated in the literature and it can be seen that they can sometimes overlap. In [3] the authors propose a code optimization which involves waveform design of the codes used, taking into account the

Manuscript received June 9, 2008; revised December 2, 2008; accepted February 25,2009 . The associate editor coordinating the review of this paper and approving it for publication was J. Andrews.

The authors are with the School of Electrical \& Electronic Engineering, The University of Manchester, PO Box 88, Manchester M16 1QD UK (e-mail: \{chris.masouros, e.alsusa\}@manchester.ac.uk).

Digital Object Identifier 10.1109/TWC.2009.080740 characteristics of the channel encountered towards achieving interuser orthogonality. Similarly, in [4] the codes are designed according to the channel in order to minimize the highest crosscorrelation amongst users. In some cases the optimized codes used are derived by a minimum mean square error (MMSE) optimization on the transmitter side, first proposed in [5]. The method proposed in [6] could be included in both the aforementioned orthogonalization mechanisms. Here, a transmitter based decorrelating technique is proposed for CDMA systems, where the spreading operation is incorporated into the decorrelating optimization. This yields a new set of codes that provide orthogonality at the receiver, again according to the channel response. In [7] the above idea is applied on MCCDMA systems and both scaled and constrained MMSE are considered. A similar approach is employed in [8], where the spreading and decorrelating procedures are unified into one block. A comprehensive study of the pre-equalization techniques on the downlink of MC-CDMA systems can be found in [9], where a similar pre-decorrelating technique is proposed. In all the above techniques spreading sequence waveform design is applied either directly or it is derived by decorrelation optimizations. Most importantly, it is done dependent on the multipath channel faced towards minimizing the crosscorrelation between the users and eliminating MAI. Code selection (instead of design) techniques for MC-CDMA are presented in [10], which again take into account the user correlations and not the instantaneous symbols, towards MAI minimization. Moreover, the codes are static throughout the frame and the selection considers the fluctuations in the channel amplitudes per subcarrier without consideration of the phase variations.

In all the approaches mentioned above, the code optimization or precoding is orientated towards eliminating all interuser interference. In this paper it is proven that there is a component of interference that contributes to the useful signal and that it could be manipulated and utilized. As a result, the application of adaptive code-to-user allocation (ACUA) on precoding schemes towards interference exploitation rather than rejection is investigated. Similarly to [10], ACUA involves a code selection optimization in which the codes available in the system are dynamically re-distributed amongst the users. However, in contrast to [10], here this is done on a symbol-by-symbol basis in the form of code- 
hopping parallel to frequency-hopping studied in the literature. In addition, instead of total interference rejection, as adopted in conventional techniques, a second objective of ACUA is to influence and exploit the constructive interference inherent in the system to deliver an enhanced SINR at the receiver. What's more, the optimization is based on both the channel response as well as the instantaneous data as they both influence the resulting instantaneous MAI. Hence, MAI can be manipulated by appropriately redistributing the codes and consequently the crosscorrelation values amongst the users taking into account the values of the data symbols to be transmitted at each symbol period. ACUA was initially introduced in [11] for DS-CDMA communications as stand-alone processing taking place at the transmitter, while signal enhancement processing was carried out at the MU receivers in the form of multi-user detection (MUD). This approach while providing performance benefits, still suffers from increased complexity MUs as MUD yields a significant computation burden and in general requires channel knowledge of all users at each MU. In this paper we modify ACUA and expand the idea further to propose the use of the dynamic partial precoding (DPP) of [12] instead as a second stage of transmit processing, shifting the signal enhancement processing complexity to the BS transmitter where computation resources as well as channel state information (CSI) for all users can be readily available. Remarkably, the simulation results shown later in this paper indicate that the performance gains comparing to the ones in [11] and [12] are further magnified when precoding - even in the form of conventional $\mathrm{ZF}$ - is employed in conjunction with ACUA. The main focus of this work is to show that by pairing ACUA with selective pre-decorrelating of the users using DPP and exploiting the constructive while suppressing the destructive component of the MAI, the SINR can be further increased and performance can be further improved. It should be noted that with this method the improvement in the received SINR is attained without the need for additional per-user-power investment at the transmitter, as energy inherent in the system is utilized.

It may be clear by now that due to ACUA the code employed by each user can vary between symbol periods. Therefore, the proposed technique entails some overhead in the form of transmitting side information (SI) to inform the MU receivers of their code allocation at each symbol period to achieve correct de-spreading. However, since the SI bits are common to all users, it will be demonstrated in the results section that the bandwidth efficiency reduction due to the SI transmission can be maintained at less than $6 \%$ of the bandwidth. This constitutes a further enhancement with respect to [11] where the efficiency reduction values reach $10 \%$. The SI bits trade-off will be shown to be worthwhile as the achieved bit error rate (BER) gains are significant compared to the non-adaptive case, especially when DPP is used.

\section{Downlink Model And Mai Characterization}

\section{A. MC-CDMA Downlink}

In order to illustrate the separation between constructive and destructive MAI a basic communication system without precoding, utilizing simple channel pre-equalization is presented.
In further analysis as well as simulations, however, the use of linear precoding is investigated to illustrate the performance boost achievable in more advanced systems. Furthermore, a partial precoding scheme is incorporated in order to optimally exploit the benefits of ACUA processing.

Consider the downlink transmission in a discrete-time synchronous frequency selective MC-CDMA system of $K$ users with spreading gain of $L$, where all codes and channels are assumed normalized to unit energy. For simplicity we assume that the number of orthogonal frequency division multiplexing (OFDM) subcarriers $M=L$. The use of a sufficiently long cyclic prefix is presumed so that intersymbol interference (ISI) is completely suppressed. For the following analysis and simulations Maximum Ratio Transmission (MRT) preequalization [2] is considered, with coefficients $\left(\mathbf{E}_{u}\right)$ given by

$$
\mathbf{E}_{u}=\mathbf{H}_{u}^{*}
$$

where $(*)$ denotes the complex conjugate. The received signal at the $u$-th MU can be expressed in matrix form as

$$
\mathbf{r}^{(i)}=\left[\mathbf{x}^{(i)} \cdot \mathbf{A} \cdot(\mathbf{C} \circ \mathbf{E})\right] \circ \mathbf{H}_{u}+\mathbf{N}_{u}
$$

where $\mathbf{x}^{(i)}=\left[\begin{array}{lll}x_{1}^{(i)} & x_{2}^{(i)} & \ldots \\ x_{K}^{(i)}\end{array}\right]$ is the $1 \times K$ vector containing all users' data for the $i$-th symbol period, $\mathbf{A}=$ $\operatorname{diag}\left(\left[a_{1} a_{2} \ldots a_{K}\right]\right)$ is the $K \times K$ diagonal matrix of amplitudes, $\mathbf{E}=\left[\begin{array}{llll}\mathrm{E}_{1} & \mathrm{E}_{2} & \ldots & \mathrm{E}_{K}\end{array}\right]^{T}$ and $\mathbf{C}=\left[\begin{array}{llll}\mathrm{C}_{1} & \mathrm{C}_{2} & \ldots & \mathrm{C}_{K}\end{array}\right]^{T}$ are $K \times L$ matrices containing the users pre-equalization coefficients and codes while $\mathbf{H}_{u}$ and $\mathbf{N}_{u}$ are the $1 \times L$ vectors representing the $u$-th MU's channel and the additive white Gaussian noise (AWGN). In (2) the expression in brackets is the transmitted signal and the notation $(0)$ is used to denote element-wise matrix multiplication. The received data for the $u$-th user and the crosscorrelation between users can be expressed as

$$
\begin{gathered}
d_{u}^{(i)}=r_{u}^{(i)} \cdot C_{u}^{H}=a_{u} \rho_{u u} x_{u}^{(i)}+\sum_{k=1, k \neq u}^{K} a_{k} \rho_{k u} x_{k}^{(i)}+n_{u}^{(i)} \\
\rho_{p q}=\left(\mathbf{C}_{p} \circ \mathbf{E}_{p} \circ \mathbf{H}_{q}\right) \circ \mathbf{C}_{q}^{H} .
\end{gathered}
$$

respectively where $\left(^{H}\right)$ denotes the Hermitian operation. In (3) $x_{u}^{(i)}$ is the desired user's signal,

$$
\sum_{k=1, k \neq u}^{K} a_{k} \rho_{k u} x_{k}^{(i)}=M A I_{u}^{(i)}
$$

is the MAI caused by the $K-1$ interferers and $n_{u}^{(i)}$ is the noise component after dispreading.

\section{B. Constructive-Destructive MAI Separation}

The instantaneous MAI in (5) can be separated to constructive and destructive components. To illustrate this we present a simple system of $K=2$ equal power users with signals $x_{1}^{(i)}=-1, x_{2}^{(i)}=1$ in Fig. 1. The vector representations of the signals for the case of orthogonal (Fig. 1a) and nonorthogonal codes (Fig 1b,c) are shown. The figure depicts the 

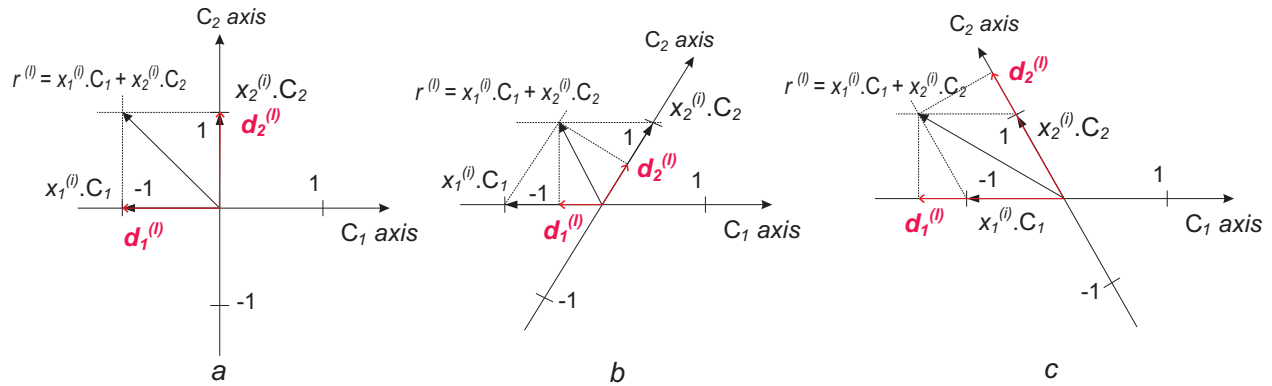

Fig. 1. Fig.1.Vector representation of the signals of $\mathrm{K}=2$ users for orthogonal (a) and non-orthogonal codes (b,c)

code axes for each case followed by the modulated $\left(x_{k}^{(i)} \cdot \mathbf{C}_{k}\right)$, the noiseless received signal $\left(r^{(i)}\right)$ and the match filtered outputs $\left(d_{k}^{(i)}\right)$. Since the subject of our investigation is MAI we assume zero noise.

For orthogonal codes (Fig. 1a), since there is no interference, the projection of the received signal $r^{(i)}$ on the code axes (match filtered output $d_{k}^{(i)}$ ) yields the exact initial symbol.

As regards non-orthogonal codes, it can be seen that the ones in Fig. 1b yield destructive interference. The addition of the spread signals in $r^{(i)}$ is destructive, the received signal has lower amplitude and the projection of $r^{(i)}$ on the codes' axes is smaller than the initial symbols. Hence, the symbol energy has been decreased. For the codes in Fig. 1c however, the magnitude of $r^{(i)}$ is increased as each spread signal adds on the other's amplitude, and therefore, the projections $d_{k}^{(i)}$ have larger magnitude than the initial symbols $x_{k}^{(i)}$. This means that the received symbol energy, due to constructive MAI has increased. Therefore, since constructive MAI adds to the useful signal's energy, the instantaneous per symbol period effective SINR can be written as

$$
S I N R_{e}=\frac{S+M A I_{\text {constructive }}}{M A I_{\text {destructive }}+N}
$$

where the power of MAI is separated to constructive and destructive components. The instantaneous interference can be characterized by observing the matrix

$$
\begin{aligned}
& M^{(i)}=\operatorname{diag}\left(x^{(i)} A\right) \cdot R \cdot \operatorname{diag}\left(x^{(i)}\right) \\
& =\left(\begin{array}{ccc}
a_{1} \rho_{11} & x_{2}^{(i)} a_{1} x_{1}^{(i)} \rho_{12} \cdots & x_{K}^{(i)} a_{1} x_{1}^{(i)} \rho_{1 K} \\
x_{1}^{(i)} a_{2} x_{2}^{(i)} \rho_{21} & a_{2} \rho_{22} \cdots & x_{K}^{(i)} a_{2} x_{2}^{(i)} \rho_{2 K} \\
\vdots & \ddots & \vdots \\
x_{1}^{(i)} a_{K} x_{K}^{(i)} \rho_{K 1} & x_{2}^{(i)} a_{K} x_{K}^{(i)} \rho_{K 2} \cdots & a_{K} \rho_{K K}
\end{array}\right)
\end{aligned}
$$

where $\mathbf{R}$ is the $K \times K$ crosscorrelation matrix

$$
R=\left(\begin{array}{lll}
\rho_{11} & \rho_{12} \cdots & \rho_{1 K} \\
\rho_{21} & \rho_{22} \cdots & \rho_{2 K} \\
\vdots & \ddots & \vdots \\
\rho_{K 1} & \rho_{K 2} \cdots & \rho_{K K}
\end{array}\right)
$$

with elements $\rho_{k u}$ as in (4). For binary phase shift keying (BPSK) modulation, the sign of the real part of each element of $\mathbf{M}^{(i)}$ provides information on whether two users interfereconstructively or destructively. By taking the sign of $\operatorname{sum}\left(\left[\operatorname{Re}\left\{\mathbf{M}^{(i)}\right\}-\operatorname{diag}\left(\operatorname{Re}\left\{\mathbf{M}^{(i)}\right\}\right)\right]_{j}\right)$ where $[.]_{j}$ denotes the $j$-th column of a matrix, one can derive whether the
cumulativeMAI component is constructive or destructive for each user, for the $i$-th symbol period of interest. Hence, the constructive MAI criterion for the cumulative MAI could be written as

$$
\operatorname{sum}\left(\left[\operatorname{Re}\left\{M^{(i)}\right\}-\operatorname{diag}\left(\operatorname{Re}\left\{M^{(i)}\right\}\right)\right]_{u}\right)>0
$$

Looking back at Fig. 1, it can be seen that while the codes in Fig. 1b derive destructive MAI for $x_{1}^{(i)}=-1, x_{2}^{(i)}=1$, they yield constructive MAI for other symbol combinations such as $x_{1}^{(i)}=1, x_{2}^{(i)}=1$ and $x_{1}^{(i)}=-1, x_{2}^{(i)}=-1$, for which the codes in Fig. 1c work destructively. Therefore, for these combinations the codes of Fig. $1 \mathrm{~b}$ perform better than the ones in Fig. 1c. Hence it would be advantageous to design an adaptive system where the codes to be used are dynamically redistributed to the users according to the symbols $\mathbf{x}^{(i)}$ under transmission at the $i$-th symbol period. This would enhance the constructive component of interference on an instantaneous basis. As a result it would lead to an increase of the instantaneous SINR per symbol period and consequently provide an average SINR boost without the need to rise the transmitted per user energy.

Indeed, it can be seen in (3) that given the CSI and data knowledge readily available at the BS the decision variables at the receiver can be pre-estimated. By selecting the appropriate code allocation for transmission at each symbol period the allocation of the factors $\rho_{k u}$ amongst the users can be influenced and hence the distribution of the $\mathbf{d}_{u}^{(i)}$ values in (3) for all users can be improved to offer enhanced reliability in the detection stage.

\section{Stage 1: Adaptive Code To User Allocation (ACUA)}

The ACUA algorithm works as shown in the diagram in Fig. 2. A number of $p_{c}$ allocation patterns are formed from an initial reference set of $N_{c}=K$ codes by randomly shuffling them amongst the users. For example, from the reference code set $\left[\mathrm{C}_{\mathrm{A}} \mathrm{C}_{\mathrm{B}} \mathrm{C}_{\mathrm{C}} \mathrm{C}_{\mathrm{D}}\right]^{T}$ in a $K=4$ user system, three possible shuffled code sets could be formed as $\left[\mathrm{C}_{\mathrm{D}} \mathrm{C}_{\mathrm{C}} \mathrm{C}_{\mathrm{A}}\right.$ $\left.\mathrm{C}_{\mathrm{B}}\right]^{T},\left[\begin{array}{lllll}\mathrm{C}_{\mathrm{B}} & \mathrm{C}_{\mathrm{D}} & \mathrm{C}_{\mathrm{A}} & \mathrm{C}_{\mathrm{C}}\end{array}\right]^{T}$ and $\left[\begin{array}{llll}\mathrm{C}_{\mathrm{C}} & \mathrm{C}_{\mathrm{A}} & \mathrm{C}_{\mathrm{B}} & \mathrm{C}_{\mathrm{D}}\end{array}\right]^{T}$ where the $k$-th row of the matrix corresponds to the code allocated to user $k$. By taking the index of each of the codes in the above sets, the allocation patterns, including the reference, could be derived as $[\mathrm{A}, \mathrm{B}, \mathrm{C}, \mathrm{D}],[\mathrm{D}, \mathrm{C}, \mathrm{A}, \mathrm{B}],[\mathrm{B}, \mathrm{D}, \mathrm{A}, \mathrm{C}]$ and $[\mathrm{C}$, $\mathrm{A}, \mathrm{B}, \mathrm{D}]$ respectively. Now the $k$-th entry in the allocation pattern denotes the index of the code intended for user $k$. At the BS transmitter, in order to choose the appropriate 


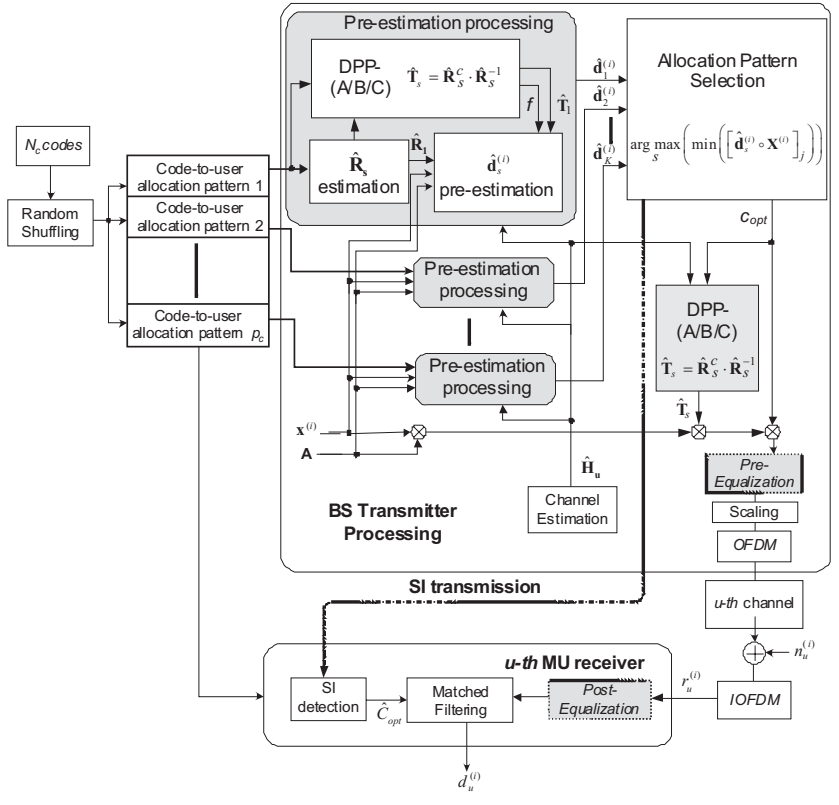

Fig. 2. Fig. 2. Flow diagram of ACUA - DPP

code allocation pattern prior to transmission, the expected decision variables at the MUs for all the available code-touser allocation combinations need to be determined using knowledge of the instantaneous symbol values for the active users as well as the CSI. Hence, in the proposed method, the estimated effective crosscorrelation matrix $\hat{\mathbf{R}}_{s}$ similar to $\mathbf{R}$ in (8) is formed for each ( $s$-th) allocation pattern at the BS from the estimated $\hat{\rho}_{k u}=\left(\mathbf{C}_{k} \circ \mathbf{E}_{k} \circ \hat{\mathbf{H}}_{u}\right) \cdot \mathbf{C}_{u}^{H}$ in which the estimated channel coefficients $\hat{\mathbf{H}}_{u}$ are used. Using this and according to the precoding employed in the system, the decision variables $\hat{\mathbf{d}}_{s}^{(i)}$ for the $s$-th code allocation can be preestimated for each of the $p_{c}$ allocation patterns. In Fig. 2 the DPP scheme is proposed for precoding but the following analysis is not restricted to it. An optimal pattern is chosen according to the selection criteria that will be presented below. The available patterns are known at both the transmitter and the receiver. Therefore, only the index $s\left(s \in\left[1, p_{c}\right]\right)$ of the allocation pattern used at each symbol-period (common for all users) needs to be conveyed to the receivers and in this way the signaling overhead can be restrained to reasonable and practical values. The signaling is done by a control signal transmitted at a different frequency/time slot as SI, as will be discussed in section 3.1 that follows. It should be noted that in order to limit the amount of SI bits needed, ACUA is performed by selecting the code-to-user pattern from a limited number of $p_{c}$ allocation patterns instead of all $N_{c} ! /\left(N_{c}-K\right)$ ! possible patterns, where $y$ ! is the factorial of $y$. By recognizing the correct pattern each MU can find the new code assigned to it for the current symbol detection.

It might appear as if the method of determining the candidate allocation patterns plays a significant role in the overall system performance. However, since the number of candidates used is much smaller than the number of all possible candidates $\left(p_{c}<<N_{c} ! /\left(N_{c}-K\right) !\right)$ it is inadequate to cover the absolute optimization for all possible data combinations and all possible interference environments. The factor that

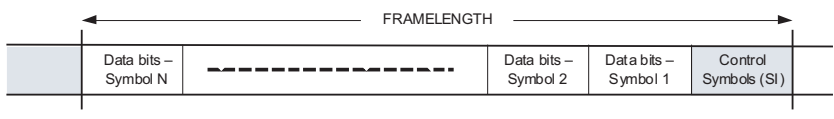

Fig. 3. Fig. 3. Frame-based transmission structure for ACUA

benefits the system is the diversity in interference introduced by forming different allocation patterns which is present as far as the candidate allocations are different and regardless of the way they are determined. Therefore it is the number of candidates $\left(p_{c}\right)$ rather than the way they are formed, that makes the difference in the resulting performance, as shown in Fig. 5 that will be discussed in the following. This is the reason for which in this contribution we have chosen to form the candidate patterns by randomly shuffling the codes amongst the users.

\section{A. Bandwidth overhead and implementation of SI transmis- sion}

It is critical to clarify that the SI involves only the index $(s)$ of the code allocation used from the $p_{c}$ available allocations, which index is universal for all users and hence the SI is common to all MUs. Consequently, it does not need to be spread and therefore introduces only a minor bandwidth overhead. The system transmission overhead is limited to $N_{s}=\left\lceil\log _{2}\left(p_{c}\right)\right\rceil$ bits for every $K$ symbols at each symbol period and can be made trivial using higher order modulation. For the example of a system with $L=32$ and $p_{c}=16$, such as the ones in the simulations that follow, assuming QPSK modulation for SI, the SI per symbol period need occupy a number of $N_{f}=N_{s} / 2=\left\lceil\log _{2}\left(p_{c}\right)\right\rceil / 2=2$ OFDM subcarriers. Presuming that one MC-CDMA-multiplexed symbol is transmitted per symbol period, the equivalent bandwidth efficiency is $L /\left(N_{f}+L\right)=94.1 \%$.

As for the timing of the SI transmission, instead of transmitting data and SI symbols in parallel, it is favorable to adopt a frame based approach as depicted in Fig. 3. Here, the allocation procedure is run for all the symbols in the frame prior to transmission and the control bits for all symbols are transmitted in the beginning of the frame separate from the data. In this way and since the SI bits are not spread they can be transmitted on separate OFDM subcarriers so that the SI transmission does not suffer from MAI. Moreover, the SI bits could be transmitted with increased power in order to make their detection more reliable and the effect to the actual data detection smaller. This is a procedure similar to the one adopted in the transmission of pilot symbols for OFDM channel estimation. It can be seen that the implementation of the SI transmission in Fig. 3 introduces some latency in the system when the frame length is large, as all the data symbols in the frame need to be processed prior to transmission. However, this simplifies the receiver processing as all the code allocations required for the coming frame are known prior to reception of the data and therefore all codes-per-symbol can be aligned to form a code stream used throughout the frame. In this way the requirement for combinatorial symbol-by-symbol processing for code adaptation at the MUs is eliminated. Moreover, in MC-CDMA, due to ISI elimination, there is no 
dependency between the data of different symbol periods and all the symbols in the frame can be processed in parallel, thus minimizing the latency penalty. The resulting complexity penalty is trivial and hence affordable at the BS, as matrix $\hat{\mathbf{R}}_{s}$ is independent of the instantaneous symbols and need not be calculated separately for each symbol period.

\section{B. ACUA selection criteria}

It is intuitive from equations (3),(7) that a number of criteria can be extracted for the selection of the optimum available code allocation pattern based on the instantaneous interference amongst users and the distribution of the resulting values in $\hat{\mathbf{d}}_{s}^{(i)}$. For reasons of clarity it should be noted that after the pre-estimation of the decision values of all the users for all available code allocation patterns, $\hat{\mathbf{d}}_{s}^{(i)}$ is a matrix of dimensions $p_{c} \times K$, where each row represents the distribution of the users' decision variables for the respective code allocation pattern. Since the performance of the worst user catalytically affects the overall system BER, the following code pattern selection criteria is proposed and examined here

$$
s_{\text {opt }}=\underset{s}{\arg \max }\left(\min \left(\left[\hat{\mathbf{d}}_{s}^{(i)} \circ \mathbf{X}^{(i)}\right]_{j}\right)\right)
$$

where $\mathbf{X}^{(i)}$ is the data matrix $\mathbf{x}^{(i)}$ repeated $p_{c}$ times vertically for all code allocations and $s_{o p t}$ is the allocation selected. According to the selected allocation pattern the final code matrix used to spread the symbols is determined as $\mathbf{C}_{o p t}=\{\mathbf{C}\}_{s_{o p t}}$ where $\{\cdot\}_{s}$ denotes the rearranging of the rows of a matrix according to sequence $s$. As regards the optimization in (10), multiplying each decision variable with the respective data results in positive elements when they have the same sign and negative when the decision variable is expected to result in a detection error. It can be seen that this criterion ignores the presence of noise in the system since there is no way to predict its instantaneous behavior. In more detail, $\min \left(\left[\hat{\mathbf{d}}_{s}^{(i)} \circ \mathbf{X}^{(i)}\right]_{j}\right)$ is the minimum value of the $j$-th row of $\hat{\mathbf{d}}_{s}^{(i)} \circ \mathbf{X}^{(i)}$ and determines the MU output that is the most prone to decision errors for each code allocation. From the $p_{c}$ available distributions of $\hat{\mathbf{d}}^{(i)}$ according to the $p_{c}$ available code allocation patterns, the optimum is chosen as the one that maximizes the minimum of $\left[\hat{\mathbf{d}}_{s}^{(i)} \circ \mathbf{X}^{(i)}\right] j$ which denotes the decision variable of the worst user at each symbol period for the $j$-th distribution of $\hat{\mathbf{d}}^{(i)}$. Hence, the code allocation selected $\left(\mathrm{s}_{\text {opt }}\right)$ is the one that delivers the highest decision variable for the worst user. In the case where two different allocation patterns offer the same minimum, the second minimum is considered and so on. Evidently, this is one BER optimization approach that favors the users that are more susceptible to errors. It should be noted that this is in contrast to the concept in [14] where it is derived that the best strategy would be to favor the best users for enhancing the average system performance. However, in our case the perception of the system is based on instantaneous observations, for which case the system performance is heavily affected by the worst user performance. By inspection of the proposed criterion, the comparison of the available code allocation patterns shows that by this optimization a favorable constructive to destructive MAI ratio is chosen which evidently delivers a higher SINR and boosts performance. By employing ACUA an advantageous MAI environment is delivered to the precoding stage, which translates to improved crosscorrelation matrices that need less decorrelation and yield more constructive elements.

The presented criterion for code selection translates differently to variable precoding schemes employed in the system, according to the decision variables provided at each case. In the following subsections, we firstly investigate the application of ACUA to conventional ZF beanforming for reasons of reference and then illustrate the proposed combination of ACUA with DPP. The decision variables are initially determined for each case and subsequently the method-specific criteria are presented.

\section{Stage 2: Transmitter Based Linear Precoding}

\section{A. $Z F$ beamfoming $(Z F)$}

For the case of linear ZF precoding $[5,7,13]$ the signal is multiplied by a full decorrelation matrix (T) prior to transmission so that MAI is suppressed. For scaled ZF it is proven in [5] that the optimal precoding matrix is given as $\mathbf{T}=f \cdot \hat{\mathbf{R}}^{-1}$ where

$$
f=\sqrt{\left(\sum_{k=1}^{K} a_{k}^{2}\right) /\left(\sum_{k=1}^{K} a_{k}^{2} R_{k, k}^{-1}\right)}
$$

is the scaling factor in order to maintain a fixed amount of transmitted power and $R_{k, k}^{-1}$ is the $k$-th diagonal element of matrix $\mathbf{R}^{-1}$. Completely MAI-free variables are delivered at the MU receivers for this case, provided that CSI is perfect. For reasons of clarity it should be noted that here the ZF precoding refers to the elimination of MAI while the MRT of (1) is used to pre-equalize the channel taps. Therefore the $\mathrm{ZF}$ precoding matrix $\mathbf{T}$ is formed assuming the MRT preequalization coefficients of (1) in the crosscorrelation elements of R. For constrained ZF the optimal precoding matrix is given as $\mathbf{T}=(\hat{\mathbf{R}}+\lambda \mathbf{I})^{-1}$ where $\mathbf{I}$ is the unitary matrix and $\lambda$ is a scalar that ensures that the average transmitted energy is equal to the one without precoding. The derivation of $\lambda$ is well explained in [5,7] and therefore will not be analyzed for the purposes of this paper. The pre-estimated decision variables for both cases of full linear precoding can be written as

$$
\hat{\mathbf{d}}_{s}^{(i)}=\mathbf{x}^{(i)} \mathbf{A} \mathbf{T}_{s} \hat{\mathbf{R}}_{s}
$$

For the decision variables optimization using ACUA only the components in (12) that are dependent on the code used (index $s$ ) can be influenced. Hence the criterion in (10) can be interpreted according to the precoding scheme used.

For scaled linear ZF precoding since $\mathbf{T}_{s} \cdot \hat{\mathbf{R}}_{s}=f_{s} \cdot \mathbf{I}$, by optimizing the code allocation amongst the users and the elements in $\hat{\mathbf{R}}_{s}$ only the scaling factor $f_{s}$ can be influenced according to (11). Hence, the optimization criterion of (10) translates to

$$
s_{\text {opt }}=\underset{s}{\arg \max }\left(f_{s}\right)
$$

In (13) apparently, $f_{s}$ does not depend on the instantaneous data and therefore the optimal code allocation $\mathbf{C}_{o p t}$ needs to be 
determined only at the beginning of the data frame in the case of a static channel. Consequently, in this case the transmitter processing and the transmission overhead imposed by the SI is drastically decreased as the code allocation procedure need not be dynamic and a single code allocation can be applied throughout the frame.

For constrained linear precoding $f_{s}=1$ and since $\mathbf{T}_{\mathbf{s}}$ is not an exact inverse of $\hat{\mathbf{R}}_{s}$, an amount of MAI remains in the system and can be influenced by ACUA to improve the received SINR. The selection criterion for constrained linear precoding translates to:

$$
s_{\text {opt }}=\underset{s}{\arg \max }\left(\min \left(\left[\left(\mathbf{x}^{(i)} \mathbf{A} \mathbf{T}_{s} \hat{\mathbf{R}}_{s}\right) \circ \mathbf{X}^{(i)}\right]_{j}\right)\right)
$$

Here, ACUA is used to manipulate the elements in $\hat{\mathbf{R}}_{s}$ and hence the MAI resulting elements in the matrix $\mathbf{Q}_{s}=\mathbf{T}_{s} \hat{\mathbf{R}}_{s}$ to enhance the reliability of the weakest user.

\section{B. Scaled dynamic partial precoding (DPP)}

Full pre-decorrelating of the users leads to wasting energy in two ways. Firstly, by cancelling interference that could contribute to the instantaneous symbols of interest, a significant potential benefit in the received SINR is lost, as the $M A I_{\text {constructive }}$ component in the nominator of (6) is zeroed. Secondly, excessive energy is spent in fully decorrelating and eliminating the elements of $\hat{\mathbf{R}}_{s}$ that derive this useful interference. Hence, low scaling factors are delivered which could be avoided by targeted partial pre-decorrelation. The above considerations lead to the utilization of DPP [12] which, additionally to the aforementioned, optimally exploits the enhanced constructive component of MAI delivered by ACUA. In DPP the precoding matrix is given as $\mathbf{T}_{s}=f_{\mathrm{s}} \mathbf{R}_{s}^{c} \hat{\mathbf{R}}_{s}^{-1}$ where $\mathbf{R}_{s}^{c}$ is the constructive crosscorrelation matrix that contains the elements of $\hat{\mathbf{R}}_{s}$ that yield constructive interference according to the observation of $\mathbf{M}^{(i)}$ at the $i$-th symbol period. Using the interference analysis in section 2, three different criteria were developed and explained in [12] to form matrix $\mathbf{R}_{s}^{c}$, according to different decorrelation strategies. The three resulting DPP sub-schemes are denoted as DPP-A, DPP-B, DPP-C below with respect to the ones in [12]. The reader is encouraged to study the aforementioned literature for further details on the construction of $\mathbf{R}_{s}^{c}$.

In all three DPP schemes the decision variables are given as in (12) where the difference is in the $f_{s}$ and $\mathbf{T}_{s}$ components. Since the DPP matrix is given as $\mathbf{T}_{s}=\mathbf{R}_{s}^{c} \hat{\mathbf{R}}_{s}^{-1}$, assuming perfect CSI, the criterion in (10) translates to:

$$
s_{\text {opt }}=\underset{s}{\arg \max }\left(\min \left(\left[\left(f_{s} \mathbf{x}^{(i)} \mathbf{A} \mathbf{R}_{s}^{c}\right) \circ \mathbf{X}^{(i)}\right]_{j}\right)\right)
$$

where $f_{s}$ and $\mathbf{R}_{s}^{c}$ depend on the specific DPP criterion used.

As a final remark on the function of the proposed scheme, it is clear from the above that the ACUA-DPP technique requires full CSI knowledge at the BS in order to correctly perform the code allocation and precoding matrix formation. We therefore propose to transmit on the time division duplex (TDD) [15] mode where the BS can estimate the channel during uplink reception and the need for the users to feed-back their CSI is eliminated.

\section{Comparative Complexity Evaluation of ACUA-DPP}

In order to investigate the complexity repercussions of the above methodology, a complexity comparison of the conventional and proposed techniques is illustrated in Table 1. In the values for total number of operations the common analysis found in the literature (e.g. [18]) is followed where only the principal factors $O($.$) are included as they are the ones that$ essentially impact on the complexity. From the comparison for DPP with and without ACUA it can be seen that a complexity increase is introduced by ACUA due to the $p_{c}$ trials between the available allocation patterns. Indeed, this increase is a consequence of the need to construct the preestimated crosscorrelation matrix $\hat{\mathbf{R}}_{s}$ for each trial. Hence, a factor of $p_{c}$ is introduced. It should be noted, though, that the complexity increase discussed involves the BS where resources are more affordable and complexity is less of a hindrance.

\section{Performance Analysis For Imperfect Si DETECTION}

\section{A. Dependency of overall BER performance on SI detection}

It is evident from the above that in the proposed scheme the SI detection catalytically affects the data detection. In this section a performance analysis is presented, taking into consideration the dependency on the imperfect SI transmission. It should be noted that this analysis is also applicable to [11] were such a study is not present. Let us assume that the probability of bit error is $P_{e}$ for the SI detection and $P_{d}$ for the data detection, in the case of error-free SI. For a number of $N_{s}$ SI bits the probability of correct SI detection becomes $P_{s}=\left(1-P_{e}\right)^{N_{s}}$. As for the data detection dependent on the SI performance, in the case of erroneous SI detection, the process becomes random and hence the correct detection has a probability of $\frac{1}{2}$. In the case of correct SI detection the correct data detection has a probability of $\left(1-P_{d}\right)$.

Following the above, the probability of success in the data detection considering the SI performance is given as

$$
P_{c}=\frac{1}{2} \cdot\left(1-\left(1-P_{e}\right)^{N_{s}}\right)+\left(1-P_{d}\right) \cdot\left(1-P_{e}\right)^{N_{s}}
$$

while the resulting probability of data error for imperfect SI detection is $P_{S I}=1-P_{c}$. It can be noted that for $P_{e}=0$ we have $P_{S I}=P_{d}$. For QPSK modulation assuming ISI elimination in MC-CDMA the error probability per SI bit $\left(P_{e}\right)$ is the flat fading QPSK bit error probability [16]

$$
P_{e}=\frac{1}{2} \cdot\left(1-\sqrt{\frac{\bar{\gamma}_{b}}{1+\bar{\gamma}_{b}}}\right)
$$

where

$$
\bar{\gamma}_{b}=\frac{E_{b}}{N_{0}} \cdot E\left(a_{f}^{2}\right)
$$

and $a_{f}$ is the amplitude of flat fading, $E_{b}$ is the energy per bit, $N_{0}$ is the noise power spectral density and $E(y)$ is the expected value of $y$. The value $P_{d}$ is dependent on the transmission scheme that ACUA is applied on. It is obvious that the error probability $P_{S I}$ is higher than $P_{d}$ for nonzero $P_{e}$, but it should be reminded that with the proposed 
TABLE I

COMPLEXITY IN NUMBERS OF OPERATIONS FOR DPP AND ACUA-DPP

\begin{tabular}{|c|c|c|c|}
\hline$\overline{D P P}$ & No. of operations & $A C U A-D P P$ & No. of operations \\
\hline construct $\hat{\mathbf{R}}_{s}$ & $O\left\{L \cdot K^{2}\right\}$ & construct $\hat{\mathbf{R}}_{s} \times p_{c}$ & $p_{c} \cdot O\left\{L \cdot K^{2}\right\}$ \\
\hline invert $\hat{\mathbf{R}}_{s}$ & $O\left\{K^{3}\right\}$ & construct $\mathbf{R}_{s}^{c} \times p_{c}$ & $p_{c} \cdot O\left\{L \cdot K^{2}\right\}$ \\
\hline construct $\mathbf{R}_{s}^{c}$ & $0\left\{L \cdot K^{2}\right\}$ & calculate scaling factor $\times p_{c}$ & $p_{c} \cdot O(K)$ \\
\hline multiply $\hat{\mathbf{R}}^{-1}{ }_{s}$ with $\mathbf{x}^{(i)}$ & $O\left(K^{2}\right)$ & invert $\hat{\mathbf{R}}_{s}$ of selected allocation pattern & $O\left(K^{3}\right)$ \\
\hline multiply with $\hat{\mathbf{R}}_{s}^{c}$ & $O\left(K^{2}\right)$ & multiply $\hat{\mathbf{R}}_{s}^{-1}$ with $\mathbf{x}^{(i)}$ & $O\left(K^{2}\right)$ \\
\hline calculate scaling factor & $O(K)$ & multiply with $\hat{\mathbf{R}}_{s}^{c}$ of selected allocation pattern & $O\left(K^{2}\right)$ \\
\hline spread & $O(K \cdot L)$ & spread with $\mathbf{C}_{o p t}$ & $O(K \cdot L)$ \\
\hline multiply with $\mathbf{E}$ & $O(K \cdot L)$ & multiply with $\mathbf{E}$ & $O(K \cdot L)$ \\
\hline despread & $O(K \cdot L)$ & despread & $O(K \cdot L)$ \\
\hline Total & $2 \cdot O\left(L \cdot K^{2}\right)+O\left(K^{3}\right)$ & Total & $2 \cdot p_{c} \cdot O\left(L \cdot K^{2}\right)+O\left(K^{3}\right)$ \\
\hline
\end{tabular}

ACUA-DPP, $P_{d}$ is severely reduced compared to conventional DPP schemes. Hence $P_{S I}$ for ACUA-DPP is still significantly less than the error probability of the respective conventional methods.

\section{B. Analysis of constructive MAI distribution for BPSK and calculation of the overall BER lower bound}

The aim of this section is to calculate a lower bound of the overall BER of the proposed scheme for BPSK modulation. To do this we assume the best case scenario where all destructive interference is removed and all constructive interference is preserved using ACUA with the DPP-B precoding [12]. We then calculate the expected value and variance of the constructive MAI for the normalized codes and channel in order to determine the upper bound of the SINR at the signal detection stage and approximate the overall probability of error lower bound using the calculated SINR upper bound. To facilitate the analysis, this section assumes unit amplitudes for all users. As a first stage, in order to determine the parameters of the pure unscaled interference, the scaling factor is omitted in the analysis. For large numbers of users, such as the ones in the presented simulations that follow, the scaling factor can be modeled as statistically independent to the data and interference. Therefore the parameters of the scaled interference can be subsequently found by multiplying with the average value of the scaling factor. It should be noted that when maintaining the constructive MAI with method DPP-B the users crosscorrelations based on MRT channel equalization in (4) are preserved unaltered at the receiver. Assuming only constructive MAI remaining at the received signal, the input to the decision stage at the receiver for BPSK can be expressed as:

$$
\begin{aligned}
\hat{x}_{u}^{(i)}= & \operatorname{Re}\left\{d_{u}^{(i)}\right\}=\operatorname{Re}\left\{\rho_{u u} x_{u}^{(i)}\right\} \\
& +\operatorname{Re}\left\{\sum_{k=1}^{U_{c}} \rho_{k u} x_{k}^{(i)}\right\}+\operatorname{Re}\left\{n_{u}^{(i)}\right\}
\end{aligned}
$$

The decision stage then simply extracts the sign of $\hat{x}_{u}^{(i)}$ to determine which of the BPSK symbols $\{-1,1\}$ was transmitted. For normalized codes and channel $\rho_{u u}=1$ for all $u$. In (19) the second term on the right side is the constructive interference and $U_{c}$ is the number of users that interfere constructively with the desired user. Fig. 4 shows the distribution of the real part of the constructive interference for the system parameters

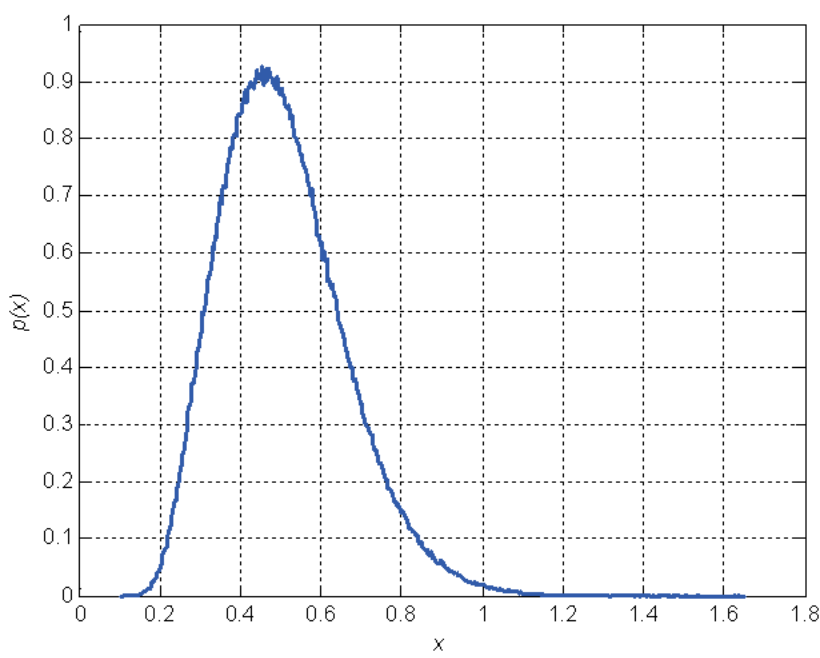

Fig. 4. Fig. 4. Probability density function of the distribution of constructive MAI for ACUA-DPP-B, for $K=32, L=32$, Rayleigh channel of $P=11, p_{c}=16$, equal power users

used in the result section $\left(K=32, L=32, P=11, p_{c}=\right.$ 16) where, for simplicity, the number of OFDM subcarriers $M=L$. The distribution graph is based on simulating $10^{6}$ instances of the communication system. It is evident that the distribution of Fig. 4 can be approximated by the Rayleigh distribution model. In what follows we calculate the mean and variance of the constructive MAI distribution.

1) Mean of constructive MAI distribution: For the interference term since the desired data $x_{u}^{(i)} \in\{-1,1\}$ is statistically independent to the interference, for the mean

of the MAI we have

$$
\begin{aligned}
E_{M A I} & =E\left\{\operatorname{Re}\left\{\sum_{k=1}^{U_{c}} \rho_{k u} x_{k}^{(i)}\right\}\right\} \\
& =E\left\{x_{u}^{(i)} \cdot \operatorname{Re}\left\{\sum_{k=1}^{U_{c}} \rho_{k u} x_{k}^{(i)}\right\}\right\} \\
& =E\left\{\operatorname{Re}\left\{\sum_{k=1}^{U_{c}} x_{u}^{(i)} \rho_{k u} x_{k}^{(i)}\right\}\right\} \\
& =\sum_{k=1}^{U_{c}} E\left\{\operatorname{Re}\left\{x_{u}^{(i)} \rho_{k u} x_{k}^{(i)}\right\}\right\}
\end{aligned}
$$

For BSPK when the interference between two individual 
users is constructive we have $\operatorname{Re}\left\{x_{u}^{(i)}\right\} \operatorname{Re}\left\{\rho_{k u} x_{k}^{(i)}\right\}>0$ and therefore $\operatorname{Re}\left\{x_{u}^{(i)} \rho_{k u} x_{k}^{(i)}\right\}=\left|\operatorname{Re}\left\{\rho_{k u}\right\}\right|$ where|.|denotes the absolute value. Therefore (20) can be rewritten as

$$
\sum_{k=1}^{U_{c}} E\left\{\operatorname{Re}\left\{x_{u}^{(i)} \rho_{k u} x_{k}^{(i)}\right\}\right\}=E\left\{U_{c}\right\} \cdot E\left\{\left|\operatorname{Re}\left\{\rho_{k u}\right\}\right|\right\}
$$

For BPSK it is shown in the appendix that $E\left\{U_{c}\right\}=$ $\frac{1}{2}(K-1)$. Therefore, in order to calculate the mean of the constructive interference distribution we need to calculate $E\left\{\left|\operatorname{Re}\left\{\rho_{k u}\right\}\right|\right\}$. Using (4) we have

$$
\begin{gathered}
E\left\{\left|\operatorname{Re}\left\{\rho_{k u}\right\}\right|\right\}=E\left\{\left|\operatorname{Re}\left\{\left(C_{u} \circ E_{u} \circ H_{k}\right) \cdot C_{k}^{H}\right\}\right|\right\} \\
=E\left\{\left|\operatorname{Re}\left\{\sum_{l=1}^{L} c_{u}^{l} \cdot c_{k}^{l *} \cdot h_{u}^{l *} \cdot h_{k}^{l}\right\}\right|\right\}
\end{gathered}
$$

For normalized real valued orthogonal codes such as the one used in this paper we have $c_{u}^{l} \cdot c_{k}^{l *}= \pm 1 / L$ and hence

$$
\begin{aligned}
E\left\{\left|\operatorname{Re}\left\{\rho_{k u}\right\}\right|\right\} \leq & E\left\{\sum_{l=1}^{L}\left| \pm \frac{1}{L} \operatorname{Re}\left\{h_{u}^{l *} h_{k}^{l}\right\}\right|\right\} \\
& =\frac{1}{L} \sum_{l=1}^{L} E\left\{\left|\operatorname{Re}\left\{h_{u}^{l *} h_{k}^{l}\right\}\right|\right\}
\end{aligned}
$$

For the normalized Rayleigh channel the complex channel coefficients (for which $E\left\{h_{u}^{l}\right\}=0, \operatorname{var}\left(h_{u}^{l}\right)=1 / L$ ) can be modeled as $h_{u}^{l}=g_{u}+i b_{u}, h_{k}^{l}=g_{k}+i b_{k}$ where $g_{m}, b_{m}$ are i.i.d. Gaussian distributed with $E\left\{g_{m}\right\}=E\left\{b_{m}\right\}=0$, $\operatorname{var}\left(g_{m}\right)=\operatorname{var}\left(b_{m}\right)=\sigma^{2}=1 /(2 L), m=u, k$. For the real part of the channel crosscorrelation in (23) we have

$$
\begin{gathered}
E\left\{\left|\operatorname{Re}\left\{h_{u}^{l *} h_{k}^{l}\right\}\right|\right\}=E\left\{\left|g_{u} g_{k}-b_{u} b_{k}\right|\right\} \\
\leq E\left\{\left|g_{u} g_{k}\right|\right\}+E\left\{\left|b_{u} b_{k}\right|\right\}
\end{gathered}
$$

For the statistically independent and uncorrelated $g_{u}, g_{k}$ we have

$$
\begin{gathered}
E\left\{\left|g_{u} g_{k}\right|\right\}=\int_{-\infty}^{\infty} \int_{-\infty}^{\infty}\left|g_{u} g_{k}\right| p\left(g_{u}\right) p\left(g_{k}\right) d g_{u} d g_{k} \\
\quad=\frac{1}{2 \pi \sigma^{2}} \int_{-\infty}^{\infty} \int_{-\infty}^{\infty}\left|g_{u}\right|\left|g_{k}\right| e^{-\left(g_{u}^{2}+g_{k}^{2}\right) / 2 \sigma^{2}} d g_{u} d g_{k}
\end{gathered}
$$

where $p\left(g_{m}\right)=e^{-\left(g_{m}^{2}\right) / 2 \sigma^{2}}$ is the probability density function (pdf) of the Gaussian distributed $g_{m}, m=u, k$. The integral in (25) represents the volume in the two-dimentional Gaussian distribution of $g_{u} g_{k}$ for which

$$
\begin{aligned}
& \int_{-\infty}^{\infty} \int_{-\infty}^{\infty}\left|g_{u}\right|\left|g_{k}\right| e^{-\left(g_{u}^{2}+g_{k}^{2}\right) / 2 \sigma^{2}} d g_{u} d g_{k} \\
& =4 \int_{0}^{\infty} \int_{0}^{\infty} g_{u} g_{k} e^{-\left(g_{u}^{2}+g_{k}^{2}\right) / 2 \sigma^{2}} d g_{u} d g_{k} \\
& =4 \int_{0}^{\infty} g_{k}\left(\int_{0}^{\infty} g_{u} e^{-\left(g_{u}^{2}+g_{k}^{2}\right) / 2 \sigma^{2}} d g_{u}\right) d g_{k} \\
& =4 \int_{0}^{\infty} g_{k}\left(-\sigma^{2} e^{-g_{k}^{2} / 2 \sigma^{2}}\right) d g_{k}=4 \sigma^{4}
\end{aligned}
$$

Therefore $E\left\{\left|g_{u} g_{k}\right|\right\}=\frac{2 \sigma^{2}}{\pi}$ and similarly $E\left\{\left|b_{u} b_{k}\right|\right\}=$ $\frac{2 \sigma^{2}}{\pi}$ which, since $\sigma^{2}=1 /(2 L)$, yields

$$
E\left\{\left|R e\left\{\rho_{k u}\right\}\right|\right\} \leq \frac{1}{L} \sum_{l=1}^{L} \frac{2}{\pi L}=\frac{2}{\pi L}
$$

for which

$$
E_{M A I} \leq \frac{K-1}{\pi L} .
$$

This is an upper bound of the expected value of the constructive MAI distribution.

2) Variance of constructive MAI distribution : Let us now compute the variance of the constructive interference term of (19). Note that the interference term can also be written as $\sum_{k=1, k \neq u}^{K} e_{k u} \rho_{k u} x_{k}^{(i)}$ where $e_{k u}=1$ if the interference between users $u, k$ is constructive and $e_{k u}=0$ otherwise. For a large number of users the crosscorrelation values can be approximated by a random Gaussian distribution and the $e k u$ variables can be modelled as Bernoulli random variables [19], independent from $\rho_{k u}$, for which $E\left\{e_{k u}\right\}=p_{e}$ and $\operatorname{var}\left(e_{k u}\right)=p_{e}\left(1-p_{e}\right)$ where $p_{e}=p\left(e_{k u}=1\right)$ is essentially the probability that two users interfere constructively. For the interference term of (19) we then have

$$
\begin{aligned}
V_{M A I} & =\operatorname{var}\left(\operatorname{Re}\left\{\sum_{k=1, k \neq u}^{K} e_{k u} \rho_{k u} x_{k}^{(i)}\right\}\right) \\
& =\sum_{k=1, k \neq u}^{K} \operatorname{var}\left(e_{k u}\right) \operatorname{var}\left(\operatorname{Re}\left\{\rho_{k u}\right\}\right) \\
& =p_{e}\left(1-p_{e}\right) \sum_{k=1, k \neq u}^{K} \operatorname{var}\left(\operatorname{Re}\left\{\rho_{k u}\right\}\right)
\end{aligned}
$$

for the crosscorrelation coefficients we have

$$
\begin{gathered}
\operatorname{var}\left(\operatorname{Re}\left\{\rho_{k u}\right\}\right)=\operatorname{var}\left(\operatorname{Re}\left\{\left(C_{u} \circ E_{u} \circ H_{k}\right) \cdot C_{k}^{H}\right\}\right) \\
=\operatorname{var}\left(\operatorname{Re}\left\{\sum_{l=1}^{L} c_{u}^{l} \cdot h_{u}^{l *} \cdot h_{k}^{l} \cdot c_{k}^{l *}\right\}\right) \\
=\operatorname{var}\left(\sum_{l=1}^{L} \pm \frac{1}{L} \operatorname{Re}\left\{h_{u}^{l *} \cdot h_{k}^{l}\right\}\right)
\end{gathered}
$$

for normalized real valued codes. For the uncorrelated Rayleigh channel (30) can be further expressed as

$$
\begin{aligned}
& \operatorname{var}\left(\sum_{l=1}^{L} \pm \frac{1}{L} \operatorname{Re}\left\{h_{u}^{l *} \cdot h_{k}^{l}\right\}\right) \\
= & \sum_{l=1}^{L} \operatorname{var}\left( \pm \frac{1}{L} \operatorname{Re}\left\{h_{u}^{l *} \cdot h_{k}^{l}\right\}\right) \\
= & \frac{1}{L^{2}} \sum_{l=1}^{L} \operatorname{var}\left(\operatorname{Re}\left\{h_{u}^{l *} \cdot h_{k}^{l}\right\}\right)
\end{aligned}
$$

Following a similar procedure to the above

$$
\begin{aligned}
\operatorname{var}\left(\operatorname{Re}\left\{h_{u}^{l *} \cdot h_{k}^{l}\right\}\right) & =\operatorname{var}\left(g_{u} g_{k}-b_{u} b_{k}\right) \\
& =\operatorname{var}\left(g_{u} g_{k}\right)+\operatorname{var}\left(b_{u} b_{k}\right)
\end{aligned}
$$

since the real and imaginary parts of the channel coefficients are statistically independent and uncorrelated. Finally, since $g_{u}, g_{k}$ are uncorrelated and have zero mean we can write 


$$
\operatorname{var}\left(g_{u} g_{k}\right)=\operatorname{var}\left(g_{u}\right) \operatorname{var}\left(g_{u}\right)=\frac{1}{4 L^{2}}
$$

which also holds for $\operatorname{var}\left(b_{u} b_{k}\right)$. Therefore (30)-(31) yield $\operatorname{var}\left(\operatorname{Re}\left\{\rho_{k u}\right\}\right)=\frac{1}{2 L^{3}}$. For the final calculation of the variance of the constructive MAI it is shown in the appendix that for BPSK we have $p_{e}=1 / 2$ and hence

$$
V_{M A I}=p_{e}\left(1-p_{e}\right) \sum_{k=1, k \neq u}^{K} \frac{1}{2 L^{3}}=\frac{K-1}{8 L^{3}}
$$

3) Lower Bound of the Probability of Error: Using to the above, the theoretical lower bound of the probability of error for the proposed scheme can be approximated by

$$
P_{d} \geq Q\left(\frac{\tilde{f} \cdot\left(E_{b}+E_{M A I}\right)}{\sqrt{V_{M A I}+N_{0} / 2}}\right)
$$

where $\tilde{f}$ is the average value of the scaling factor, $E_{b}$ is the energy per bit, $\sigma_{n}^{2}=N 0 / 2$ is the AWGN variance and $Q($. is the Q-function. Using (28) and (33) we have

$$
P_{d} \geq Q\left(\frac{\tilde{f} \cdot\left(E_{b}+\frac{K-1}{\pi L}\right)}{\sqrt{\frac{K-1}{8 L^{3}}+N_{0} / 2}}\right)
$$

It should be noted that the symbol-by-symbol redistribution of codes with ACUA as well as the adaptive nature of DPP cause fluctuations of the elements in $R_{s}^{c}$ and hence in the precoding matrix. Therefore it is not possible to calculate the average value of the scaling factor analytically. The theoretical lower bound of (36) using an empirically estimated average of the scaling factor has been added in Fig. 7 for comparison to the simulated performance.

\section{NumericAl ANd Simulation REsults}

StateMonte Carlo simulations for various combinations of ACUA with precoding have been performed. In the simulations scaled and constrained $\mathrm{ZF}$ (denoted as $\mathrm{ZF}$ and $\mathrm{cZF}$ respectively) is covered but the work is mainly focused on the combination of ACUA with DPP in order to optimally benefit from the useful MAI. For the data symbols, unless stated otherwise, BPSK modulation is employed and WalshHadamard codes of $L=32$ have been used. The multipath channel considered here is a chip-spaced Rayleigh frequency selective fading of $P=11$ resolvable paths with unity gain and equal average power per path (uniform channel power-profile). As in the mathematical analysis presented above, the use of cyclic prefix is presupposed so that the ISI is completely suppressed. As regards channel equalization, in this contribution we focus on transmitter based channel equalization, in the form of MRT, combined with precoding to completely remove the need for channel estimation at the downlink receivers and attain simplified MUs. In all simulations it is assumed that the number of OFDM subcarriers $M=64$. The SI transmission is incorporated in the simulations and non-ideal detection is considered. For reasons of bandwidth efficiency the SI bits were not spread but only QPSK modulated and transmitted with power increased by a factor of two compared to the data symbols. Hence flat fading QPSK performance is attained for

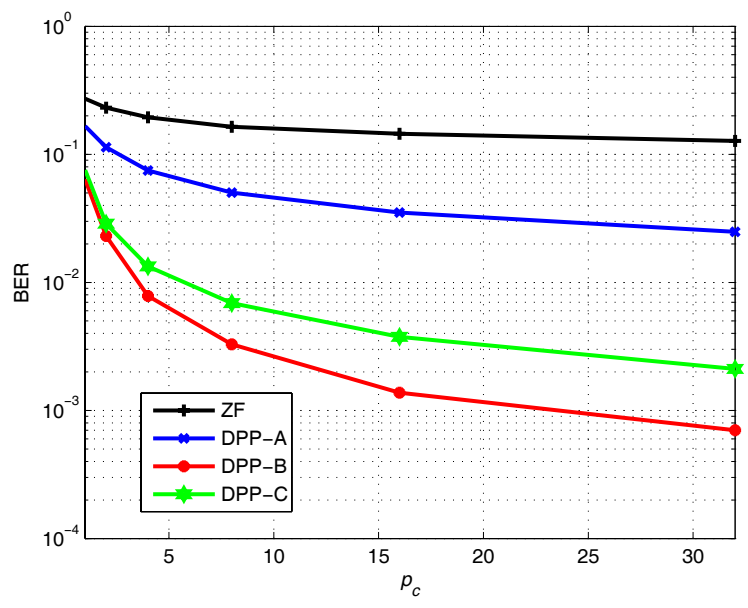

Fig. 5. Fig. 5. BER vs. $p_{c}$ performance for ACUA with ZF, DPP for a Rayleigh channel of $P=11, K=32, L=32$ at $\mathrm{SNR}=10 \mathrm{~dB}$, equal powers

the SI which provides as a lower bound for the overall system BER.

\section{A. Selection of the optimum $p_{c}$ value}

As $p_{c}$ increases, the variety in the distributions of the crosscorrelations amongst the users rises, interference can be manipulated more efficiently and the system performance optimization is enhanced. On the other hand, for increasing values of $p_{c}$ the required SI bits to be transmitted also increase and hence the transmission efficiency decreases. In order to select the optimum value of $p_{c}$ for the following simulations, a compromise between performance improvement and the amount of SI to be transmitted should be reached. For this reason the performance of ACUA on the ZF, and DPP methods to be presented for the system under consideration with $K=32, L=32$ was investigated for increasing $p c$ in fig. 5. It can be seen that all the techniques under examination show performance benefits for values of up to $p_{c}=16$ and little improvement onwards. Therefore, in order to limit the transmission overhead, a number of $p_{c}=16$ candidate code allocation patterns are used to select from, for the code allocation optimization in all the simulations that follow. For these values of $p_{c}$ and $L$, a symbol transmission rate efficiency of $L /\left(L+N_{f}\right)=32 / 34=94.1 \%$ is attained as shown in section 3.2. Although a $6 \%$ efficiency reduction is not insignificant, it will be shown that it is worthwhile as the proposed method produces a considerable BER improvement. Using a larger number of users or higher order modulation would further reduce the efficiency loss. Moreover, for scaled $\mathrm{ZF}$, as mentioned, the code allocation need not be changed at each symbol period, which dramatically reduces the SI overhead as a number of $N_{s}$ SI bits need to be transmitted per frame. For the frame length of $F=10$ symbols that is used in the following simulations the transmission rate efficiency is $(F \times L) /\left(F \times L+N_{f}\right)=(10 \times 32) /(10 \times 32+2)=99.3 \%$.

\section{B. ACUA with full linear ZF precoding}

Fig. 6 depicts the performance of ACUA with ZF and cZF. A system of $K=32$ users with codes of length $L=32$ 


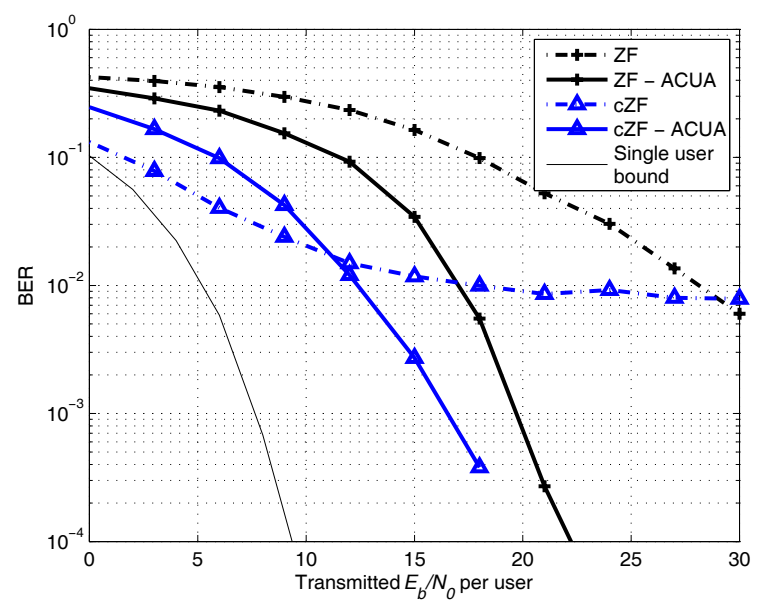

Fig. 6. Fig. 6. BER vs. SNR performance for ZF, cZF with and without ACUA, $N_{c}=K, p_{c}=16$, Rayleigh channel of $P=11$, full load $K=32, L=32$, equal powers

is considered and the ACUA parameters are fixed at $p_{c}=16$ patterns and $N_{c}=K$. The figure also includes the generic AWGN single user bound as a benchmark reference. An SNR gain of $11 \mathrm{~dB}$ can be seen for $\mathrm{ZF}$ in the area of $\mathrm{BER}=10^{-2}$. Significantly higher values of $f_{s}$ are delivered, which brings considerable increase in the resulting SINR that justifies the vast performance improvement viewed. For cZF the results show that the error floor due to MAI is eliminated when ACUA is applied since the remaining interference is constructively utilized. However, it should be observed that for low transmitted SNR values ACUA provides worse performance. This is due to the SI detection which for low SNRs is unreliable. For higher SNR values the application of ACUA clearly offers substantial benefits to the system performance.

\section{ACUA with dynamic partial precoding (DPP)}

In Fig. 7 the BER vs. SNR performance of the proposed hybrid ACUA with DPP is depicted for $K=32, L=32$, $N c=K, p_{c}=16$. Performance of ZF as well as DPP without ACUA is also shown for comparison. For DPP-B-ACUA the performance for error-free SI detection is also included to illustrate the effect of unreliable SI. It can be seen in the figure that for DPP without ACUA the selectivity in precoding on its own [12] benefits the system and provides a performance gain compared to ZF. However, further significant performance gains are achieved when ACUA is employed. For all DPP sub-techniques a transmitted SNR per user gain of 10 to 12 $\mathrm{dB}$ is attained at the BER $=10^{-2}$ level. At $E_{b} / N_{0}=12 \mathrm{~dB}$ a BER improvement of two orders of magnitude can be observed between DPP-B and DPP-B-ACUA. As regards the effect of the SI detection performance, it is evident in the graphs for DPP-B-ACUA that there is an overall performance loss for low SNRs due to poor SI reliability. However, the graphs for error-free and realistic SI converge for $\mathrm{SNR}=9 \mathrm{~dB}$ as the SI becomes more reliable. Finally, the theoretical probability of error lower bound of section 5.2.3 also appears in the figure which shows a close match to the simulated performance of DPP-B-ACUA.

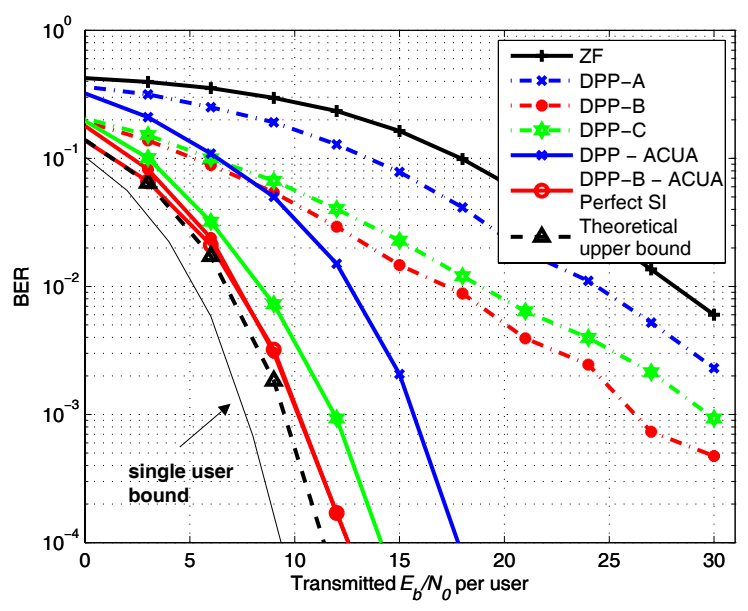

Fig. 7. Fig. 7. BER vs. SNR performance for DPP with and without ACUA, $N_{c}=K, p_{c}=16$, Rayleigh channel of $P=11$, full load $K=32, L=32$, equal powers

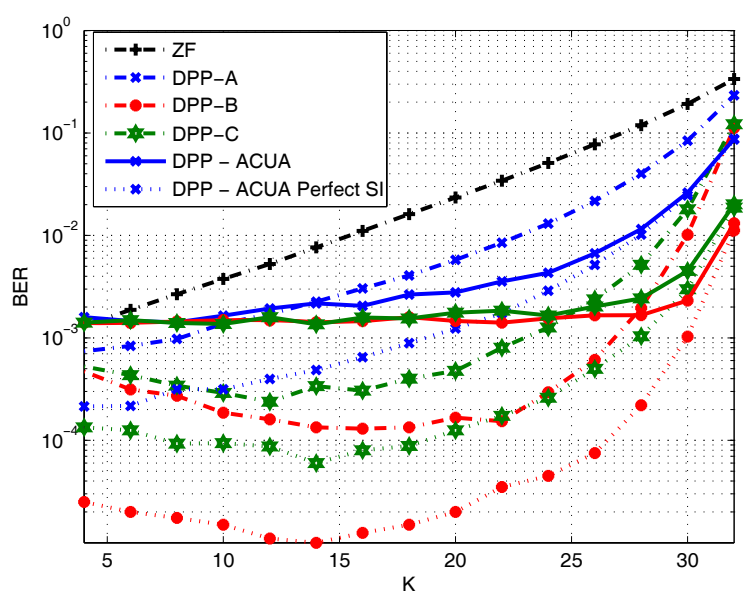

Fig. 8. Fig. 8. BER vs. $K$ performance for DPP with and without ACUA, $N_{c}=K, p_{c}=16$, Rayleigh channel of $P=11, \mathrm{SNR}=7 \mathrm{~dB}, L=32$, equal powers

In Fig. 8 the BER vs. $K$ performance of the proposed scheme is investigated for $L=32$ and transmitted $\mathrm{SNR}=7 \mathrm{~dB}$. $p_{c}$ is increased for increasing $K$ up to a maximum value of $p_{c}=16$ for $K=25$ and kept constant onwards. For the ideal case of error free SI transmission the results show a significant BER improvement for all values of $K$. However for realistic SI detection the results show that for low $K$ the proposed technique offers worse performance. This is because DPP performance is adequate even without ACUA and the SI detection imposes a lower BER bound of more than 10-3 for this SNR value when ACUA is employed. The graphs for DPP and DPP-ACUA cross over at $K=13$ for DPP-A, $K=28$ for DPP-B and DPP-C.

The simulations so far have considered only BPSK modulation. To verify the superiority of DPP-ACUA compared to DPP for higher order modulation, Fig. 9 shows the symbol error rate (SER) vs SNR performance of the proposed technique for the same scenario as in Fig. 7 of $K=32, L=32, p_{c}=16$, $P=11$ with QPSK modulation. It can be seen that the proposed DPP-ACUA still provides a significant performance benefit compared to DPP which translates to a $12 \mathrm{~dB}$ gain 


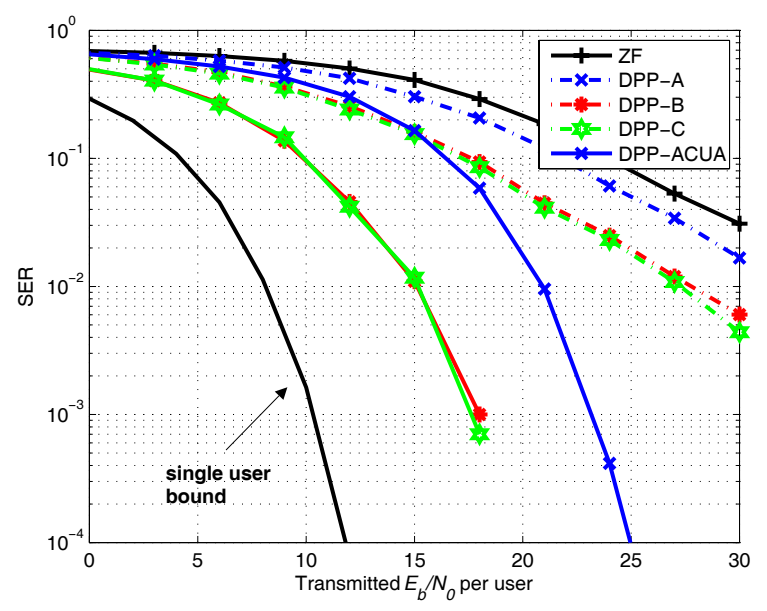

Fig. 9. Fig. 9. SER vs. SNR performance for DPP for $\mathrm{K}=32, L=32, p_{c}=16$, Rayleigh channel of $P=11$, QPSK

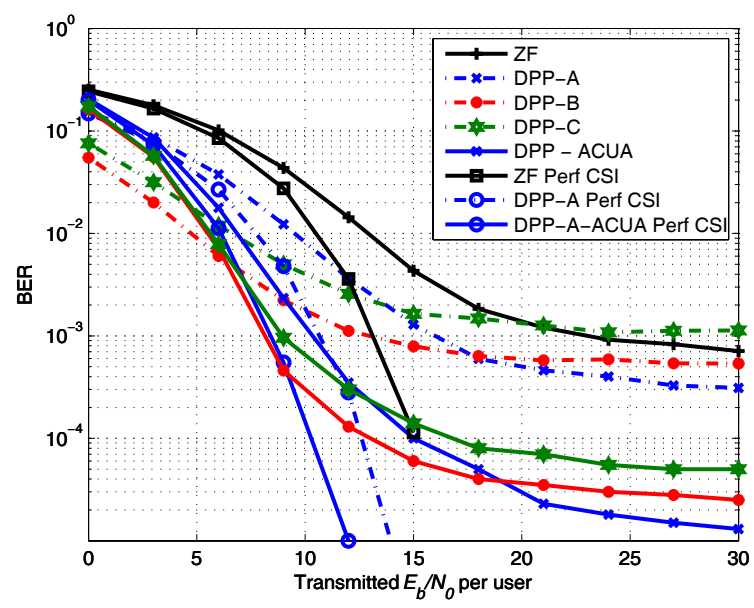

Fig. 10. Fig. 10. BER vs. SNR performance for DPP for $20 \%$ errors in CSI, $K=25, L=32$, Rayleigh channel of $P=11$, equal powers

between DPP-B -ACUA and DPP-B in the area of SER $=10^{-2}$.

In Fig. 10 the case where imperfect CSI is available at the BS transmitter is investigated. A system of $K=25, L=32$ employing BPSK is simulated and it is assumed that there is an average deviation in both real and imaginary parts of the estimated channel coefficients of $20 \%$ compared to their actual values. Results for perfect CSI are also included for ZF, DPP-A and DPP-A-ACUA to illustrate the repercussions on the system performance. As a first notation it can be seen that for low SNR values up to $7 \mathrm{~dB}$ the DPP-ACUA methods are outperformed by simple DPP. This is because the SI transmission is now problematic due to the CSI errors. However the trend shown in all the results above is re-established for higher SNRs where the proposed combination provides the best performance. Overall it can be seen that all techniques shown are considerably affected by this relatively high CSI error percentage but the proposed DPP-ACUA combination still outperforms conventional precoding and DPP for high SNR values.

\section{CONCLUSION}

In cellular MC-CDMA communication systems interference amongst the users is always present due to the multipath channel distortion. This interference can be separated to constructive and destructive. Moreover, at the downlink transmission due to knowledge of all users' data symbols, the interference can be manipulated by reallocating the codes to the users with ACUA. The constructive component of the MAI can be exploited to deliver more reliable decision variables at the MU receivers. It was shown here that by combining ACUA with DPP major BER performance benefits can be achieved.

By "fine tuning" the users' codes so that the resulting decision variables at the MUs are optimized, we have shown that the proposed technique can significantly improve performance with no need for additional power-per-user investment. The trade-off is the need for transmission of control signals which imposes a small overhead on the system efficiency.

\section{APPEndiX: Probability Of Constructive INTERFERENCE FOR BPSK MODULATION}

For a generic MRT system with $K-1$ interfering users the average number of users interfering constructively with the desired signal is $E\left\{U_{c}\right\}=p_{e} \cdot(K-1)$ where $p_{e}$ is the probability of two individual users interfering constructively. For BPSK, as shown above we have

$$
\begin{aligned}
p_{e}= & P\left(\operatorname{Re}\left(x_{u}^{(i)}\right) \cdot \operatorname{Re}\left(\rho_{k u} x_{k}^{(i)}\right)>0\right) \\
= & P\left(x_{u}^{(i)}=1, \operatorname{Re}\left(\rho_{k u} x_{k}^{(i)}\right)>0\right) \\
& +P\left(x_{u}^{(i)}=-1, \operatorname{Re}\left(\rho_{k u} x_{k}^{(i)}\right)<0\right) .
\end{aligned}
$$

Since the users' data sources and the crosscorrelations are statistically independent the first component of the right part of (37) can be written as

$$
\begin{gathered}
P\left(x_{u}^{(i)}=1, \operatorname{Re}\left(\rho_{k u} x_{k}^{(i)}\right)>0\right) \\
=P\left(x_{u}^{(i)}=1\right) \cdot P\left(\operatorname{Re}\left(\rho_{k u} x_{k}^{(i)}\right)>0\right) .
\end{gathered}
$$

For i.i.d. BPSK symbols $x_{u}^{(i)}$ we have $P\left(x_{u}^{(i)}=1\right)=1 / 2$ and for the MAI we have

$$
\begin{gathered}
P\left(\operatorname{Re}\left(\rho_{k, u} x_{k}^{(i)}\right)>0\right)=P\left(\operatorname{Re}\left(\rho_{k, u}\right) \cdot x_{k}^{(i)}>0\right) \\
=P\left(\operatorname{Re}\left(\rho_{k, u}\right)>0, x_{k}^{(i)}=1\right)+P\left(\operatorname{Re}\left(\rho_{k, u}\right)<0, x_{k}^{(i)}=-1\right)
\end{gathered}
$$

Since the data is independent of the channel. For an uncorrelated Rayleigh fading channel, the real and imaginary pars of the channel tap coefficients and hence the crosscorrelation values can be positive or negative with equal probability. Thus we get

$$
\begin{aligned}
& P\left(\operatorname{Re}\left(\rho_{k, u}\right)>0, x_{k}^{(i)}=1\right) \\
= & P\left(\operatorname{Re}\left(\rho_{k, u}\right)>0\right) \cdot P\left(x_{k}^{(i)}=1\right)=\frac{1}{2} \cdot \frac{1}{2}=\frac{1}{4}(40)
\end{aligned}
$$


By substituting back in the above equations and using the symmetry in the components of $p_{e}$ shown in (37) and (39) we find $p_{e}=\frac{1}{2}$ which yields $E\left\{U_{c}\right\}=\frac{1}{2}(K-1)$.

\section{ACKNOWLEDGMENT}

The authors would like to gratefully acknowledge the financial support for this work by EPSRC and Philips Research Labs UK, and express their thanks to Dr. Tim Moulsley for his invaluable advice.

\section{REFERENCES}

[1] S. Kaiser, Multi-Carrier CDMA Mobile Radio Systems-Analysis and Optimization of Detection, Decoding, and Channel Estimation. Dusseldorf, Germany: VDI-Verlag, 1998.

[2] I. Cosovic, Uplink Multi-Carrier CDMA Mobile Radio Systems. Belgrade: Mediagraf, 2005.

[3] G. W. Wornell, "Spread-signature CDMA: efficient multiuser communication in the presence of fading," IEEE Trans. Inform. Theory, vol. 41, pp. 1418-1438, Sept. 1995.

[4] W. H. Mow, "Minimizing the worst-case interuser interference experienced by any user in CDMA systems: a metric approach," in Proc. Int. Symp. Spread-Spectrum Techniques Applications, vol. 2, Mainz, Germany, Sept. 1996, pp. 561-565.

[5] B. R. Vojcic and W. M. Jang, "Transmitter precoding in synchronous multiuser communications," IEEE Trans. Commun., vol. 46, no. 10, pp. 1346-1355, Oct. 1998.

[6] M. Brandt-Pearce and A. Dharap, "Transmitter-based multiuser interference rejection for the down-link of a wireless CDMA system in a multipath environment," IEEE J. Select. Areas Commun., vol. 18, no. 3, pp. 407-417, Mar. 2000.

[7] M. Morelli and L. Sanguiletti, "A novel prefiltering technique for downlink transmissions in TDD MC-CDMA systems," IEEE Trans. Wireless Commun., vol. 4, no. 5, Sept. 2005.

[8] I. Cosovic, S. Sand, and R. Raulefs, "A non-linear precoding technique for downlink MC-CDMA," in Proc. IEEE Veh. Technol. Conf. Spring 2005, vol. 3, pp. 1711-1715, 2005.

[9] P. Bisanglia et al., "Pre-equalization techniques for downlink and uplink TDD MC-CDMA systems," Int. J. Wireless Personal Commun., vol. 35, no. 1-2, pp. 3-18, Oct. 2005.

[10] S. Nobilet, J.-F. Helard, and D. Mottier, "Spreading sequences for uplink and downlink MC-CDMA systems: PAPR and MAI minimization," European Trans. Telecommun., vol. 13, no. 5, pp. 465-473, 2002.

[11] E. Alsusa and C. Masouros, "Adaptive code allocation for interference management on the downlink of DS-CDMA systems," IEEE Trans. Wireless Commun., vol. 7, no. 7, July 2008.
[12] C. Masouros and E. Alsusa, "A new dynamic partial precoding technique for MC-CDMA systems employing PSK modulation," in Proc. MultiCarrier Spread Spectrum Workshop, May 2007, pp. 177-186.

[13] Papathanasiou, M. Meurer, T. Weber, and P. W. Baier, "A novel multiuser transmission scheme requiring no channel estimation and no equalization at the mobile stations for the downlink of TD-CDMA operating in the TDD mode," in Proc. IEEE Veh. Technol. Conf. Fall 2000, pp. 203-210.

[14] R. Knopp and P. Humblet, "Information capacity and power control in single-cell multiuser communications" in Proc. IEEE International Conf. Commun. (ICC) vol. 1, Seatle, June 1995, pp. 331-335.

[15] R. Esmailzadeh and M. Nakagawa, TDD-CDMA for Wireless Communications. London: Artech House, 2002.

[16] J. G. Proakis, Digital Communications. McGraw-Hill, 3rd ed., 1995.

[17] S. Verdu, Multiuser Detection. Cambridge University Press, 1998.

[18] M. G. Alkhansari and A. B. Gershman, "Fast antenna subset selection in MIMO systems," IEEE Trans. Signal Processing, vol. 52, no. 2, pp. 339-347, Feb. 2004.

[19] A. Leon-Garcia, Probability and Random Processes for Electrical Engineering. Reading, MA: Addison-Wesley, 1994.

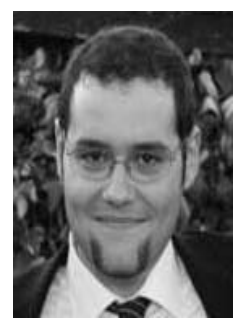

Christos Masouros (M'06), received his Diploma in Electrical \& Computer Engineering from the University of Patras, Greece in 2004 and the MSc by research and $\mathrm{PhD}$ degrees in Electrical \& Electronic Engineering from the University of Manchester, UK in 2006 and 2009 respectivelly. He is currently a Research Associate at the Microwave and Communication Systems group of the University of Manchester. His research interests lie in the field of wireless communications and signal processing with particular focus on interference mitigation techniques for CDMA, multicarrier and MIMO communications

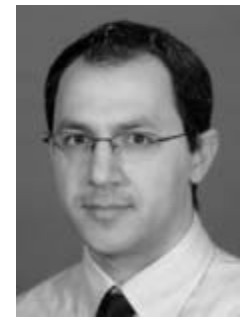

Emad Alsusa (M'06-SM'07), received BSc in Electrical \& Electronic Engineering from Salford University, Salford, UK, in 1996 and $\mathrm{PhD}$ in Electrical \& Electronic Engineering from Bath University, Bath, UK, in 2000. From June 2000 to September 2003 he was with the School of Engineering and Electronics at Edinburgh University as a Postdoctoral Research fellow. He joined Manchester University in 2003 as a Lecturer of Communication Engineering. $\mathrm{He}$ is a senior member of the IEEE. His research interests are in the area of wireless communication networks, especially, modulation and multiple access techniques, channel estimation, channel coding, interference mitigation, multiuser detection and MIMO techniques. 